\title{
A importância do farmacêutico no acompanhamento de pacientes hipertensos
}

\author{
The importance of the pharmacist in monitoring hypertensive patients \\ La importancia del farmacéutico en el seguimiento de los pacientes hipertensos
}

Recebido: 08/04/2021 | Revisado: 14/04/2021 | Aceito: 31/07/2021 | Publicado:06/08/2021

\author{
Vilcilene Nascimento Diniz de Sousa \\ ORCID: https://orcid.org/0000-0001-7152-2701 \\ Faculdade Pitágoras, Brasil \\ E-mail: vilcilenediniz@gmail.com \\ Geise Raquel Sousa Pinto \\ ORCID: https://orcid.org/0000-0002-6444-7800 \\ Laboratório Municipal do Hospital Dom Hélio Campos, Brasil \\ E-mail: geise.raquel@ hotmail.com
}

\begin{abstract}
Resumo
Nos últimos anos o envelhecimento da população tem sido considerado algo de grande preocupação para a saúde pública, visto que com o envelhecimento da população as doenças crônicas não transmissíveis tem ocupado um lugar de destaque no contexto da saúde pública do Brasil. A hipertensão arterial tem se tornado uma doença mais frequente nos últimos anos, acometendo principalmente pacientes da terceira idade. Mesmo com ações e projetos para diminuir os riscos da incidência da hipertensão diversos fatores podem estar associados ao aparecimento da doença, sendo necessário acompanhamento pelo um profissional qualificado. O problema desse trabalho é: Quais os possíveis problemas relacionados ao uso de medicamentos por pacientes hipertensos? O objetivo geral deste trabalho é abordar a importância da atenção farmacêutica para pacientes hipertensos. A metodologia empregada nesse trabalho foi uma pesquisa bibliográfica reunida por meio de pesquisas monográficas, artigos, revistas, periódicos e livros relacionados ao tema. Como resultado deste trabalho, pode-se afirmar que o farmacêutico é um profissional de extrema importância para o acompanhamento farmacoterapêutico de um paciente hipertenso, visto também ser o profissional mais próximo dos usuários em farmácias e drogarias. O acompanhamento do farmacêutico possibilita melhora no tratamento e continuidade com o uso racional do medicamento.
\end{abstract}

Palavras-chave: Hipertensão; Medicamentos; Atenção farmacêutica.

\begin{abstract}
In recent years, the aging of the population has been considered something of great concern for public health, since with the aging of the population, chronic non- communicable diseases have occupied a prominent place in the context of public health in Brazil. Arterial hypertension has become a more frequent disease in recent years, affecting mainly elderly patients. Even with actions and projects to reduce the risks of the incidence of hypertension, several factors may be associated with the onset of the disease, requiring monitoring by a qualified professional. The problem with this work is: What are the possible problems related to medication use by hypertensive patients? The general objective of this work is to address the importance of pharmaceutical care for hypertensive patients. The methodology used in this work was a bibliographic research gathered through monographic research, articles, magazines, periodicals and books related to the theme. As a result of this work, it can be stated that the pharmacist is an extremely important professional for the pharmacotherapeutic follow-up of a hypertensive patient, since he is also the closest professional to users in pharmacies and drugstores. The pharmacist's follow- up enables improvement in treatment and continuity with the rational use of the medication.
\end{abstract}

Keywords: Hypertension; Medicines; Pharmaceutical attention.

\section{Resumen}

En los últimos años, el envejecimiento de la población ha sido considerado algo de gran preocupación para la salud pública, ya que con el envejecimiento de la población, las enfermedades crónicas no transmisibles han ocupado un lugar destacado en el contexto de la salud pública en Brasil. La hipertensión arterial se ha convertido en una enfermedad más frecuente en los últimos años, afectando principalmente a pacientes de edad avanzada. Incluso con acciones y proyectos para reducir los riesgos de la incidencia de hipertensión, varios factores pueden estar asociados con la aparición de la enfermedad, requiriendo un seguimiento por parte de un profesional calificado. El problema de este trabajo es: ¿Cuáles son los posibles problemas relacionados con el uso de medicamentos por parte de pacientes hipertensos? El objetivo general de este trabajo es abordar la importancia de la atención farmacéutica para los pacientes hipertensos. La metodología utilizada en este trabajo fue una investigación bibliográfica recopilada a través de investigaciones monográficas, artículos, revistas, publicaciones periódicas y libros relacionados con la temática. Fruto de este trabajo, se puede decir que el farmacéutico es un profesional de suma importancia para el seguimiento 
farmacoterapéutico de un paciente hipertenso, ya que también es el profesional más cercano a los usuarios en farmacias y droguerías. El seguimiento del farmacéutico permite la mejora del tratamiento y la continuidad con el uso racional de la medicación.

Palabras clave: Hipertensión; Medicamentos; Atención farmacéutica.

\section{Introdução}

Com o envelhecimento da população as doenças crônicas têm passado a ocupar um lugar de destaque de modo que os pacientes geriátricos comumente necessitam fazer uso de uma quantidade significativa de medicamentos para curar ou controlar as doenças típicas dessa faixa etária, que muitas vezes são favorecidas pelo processo de envelhecimento (Pereira et al., 2015). A hipertensão arterial (HA) é uma das cardiopatias que mais causam complicações clínicas e óbitos, tornando-se a doença de maior prevalência no mundo moderno (Mion; Pierin \& Guimarães, 2001). Em estudos de metanálise realizados do Brasil, entre os anos de 1980 a 2000, foi estimada uma prevalência desta patologia em 31\% da população geral do país (Coelho et al., 2019).

A Atenção Farmacêutica (AF) é um conjunto de ações que são desenvolvidas pelo profissional farmacêutico, que também compreende atitudes, valores éticos, comportamentos, habilidades, compromissos e corresponsabilidades na prevenção de doenças, promoção e recuperação da saúde, de forma integrada à equipe de saúde. É uma interação direta do farmacêutico com o usuário, visando a uma farmacoterapia racional, e à obtenção de resultados definidos e mensuráveis, voltados para a melhoria da qualidade de vida com interação biopsicossocial, sob a ótica da integralidade das ações de saúde (Brasil, 2002).

Quais os possíveis problemas relacionados ao uso de medicamentos por pacientes hipertensos? A utilização de medicamentos é uma condição comum entre a população em geral, o que tem ocasionado sérios problemas, levando em conta a alta prevalência do uso indevido, seja devido à prescrição, à dispensação ou à utilização dos mesmos. A Organização Mundial de Saúde (OMS), acredita que mais de 50\% dos medicamentos são prescritos ou dispensados de forma errada e que $50 \%$ dos pacientes tomam medicamentos de maneira incorreta aumentado o índice de morbidade e mortalidade (Marin; Santos, 2016).

No paciente com hipertensão arterial sistêmica (HAS) o acompanhamento farmacoterapêutico é imprescindível a fim de minimizar os riscos com os problemas relacionados aos medicamentos, promovendo educação em saúde, o que é parte fundamental da Atenção Farmacêutica (Oliveira et al., 2020).

Esse trabalho teve como objetivo geral abordar a importância da atenção farmacêutica para pacientes hipertensos. O papel do farmacêutico contempla informações quanto ao uso correto dos medicamentos, acondicionamento das drogas, duração do tratamento e a avaliação da prescrição, visando a não ocorrência de viés que possam prejudicar a saúde ou o tratamento do paciente, bem como o controle dos riscos, de modo a se evitar possíveis morbidades e/ou mortalidade (Souza; Bertoncin, 2008) e como objetivos específicos descrever a fisiopatologia da hipertensão arterial sistêmica; Discutir o tratamento da hipertensão, bem como os possíveis problemas relacionados ao uso de medicamentos nos pacientes hipertensos; Apontar a importância do farmacêutico para pacientes hipertensos.

\section{Metodologia}

Assim, este trabalho trata-se de uma revisão bibliográfica com abordagem qualitativa e descritiva, sobre a Importância do Acompanhamento Farmacoterapêutico em Pacientes Hipertensos. A pesquisa foi feita nas bases de dados de sites científicos: Scientific Eletronic Library Online-SciELO, Medline/Pubmed, Google acadêmico, Literatura Latino Americana e do Caribe em ciências da saúde (LILACS), livros, revisitas, acervos disponíveis em sites de universidades. O critério para escolha do estudo será de publicações com teor científico a fim de coletar informações acerca do tema, com delimitação no tempo de 2010 a 2020. Para a busca foram utilizadas as palavras-chave: Hipertensão, medicamentos, atenção farmacêutica. 


\section{Resultados e Discussão}

A pressão é uma força motriz que produz distensão no seu continente e movimentação do seu conteúdo. Pressão é igual à força sobre área $\mathrm{P}=\mathrm{F} / \mathrm{A}$. No nosso caso pressão hidrostática dos líquidos ou do sangue é regido por leis físicas bem definidas. Assim, a pressão do sangue gerado pela força motriz que é o coração (bomba) dentro das nossas artérias define a distensão da sua parede (tensão) e a velocidade com que o sangue percorre estes vasos chamados de condutância (maiores) e resistência (menores). O sangue se movimenta de forma continua graças as diferenças de pressões deste circuito fechado também conhecido como pressurizado. A maior pressão em estado fisiológico em repouso é de $120 \mathrm{mmHg}$, e ocorre durante a ejeção ventricular e vai percorrendo a arvore arterial em direção a valores de pressão menores (gradiente) até valores de $80 \mathrm{mmHg}$ que corresponde a pressão diastólica definida pela resistência das meta-arteríolas em condições normais (Silva; Oliveira; Pierin, 2016).

De acordo com a Sociedade Brasileira de Cardiologia (2016) o conceito de Hipertensão Arterial (HA), descrito na $7^{\text {a }}$ Diretriz Brasileira de HA é definido como: Uma condição clínica em que os níveis pressóricos estão na grande maioria das vezes elevados, $\geq 140$ e/ou $90 \mathrm{mmHg}$ produzindo de forma regular lesão das artérias de grande, médio e pequeno calibre, bem como lesão a nível de coração de diversas formas e de outros órgãos importantes como cérebro e rins. Órgãos estes, que recebem maior parte do fluxo sanguíneo. Além destas modificações físicas de desgastes por remodelamento vascular/ hipertrofia e hipertrofia miocárdica da mesma forma a HAS se associa com variações metabólicas podendo ser piorado pelos fatores de risco como: dislipidemia, obesidade, tabagismo, estresse psicoemocional, diabetes mellitus (DM), obesidade visceral e história familiar de HAS e outras patologias cardiovasculares precoce na família. Essa condição se associa de forma robusta, independente e consistente com eventos como morte súbita, acidente vascular encefálico (AVE), infarto agudo do miocárdio (IAM), insuficiência cardíaca (IC), doença arterial periférica (DAP) e doença renal crônica (DRC), fatal e não fatal (Malachias et al., 2016).

O conceito de pré-hipertensão descrito na $7^{\mathrm{a}}$ Diretriz Brasileira de Hipertensão Arterial é definido como: Uma condição caracterizada por pressão arterial sistólica (PAS) entre 121 e 139 e/ou PA diastólica (PAD) entre 81 e 89 mmHg (Malachias et al., 2016). Há uma ligação direta e linear entre envelhecimento e prevalência de HA, relacionada ao: Aumento da expectativa de vida da população brasileira, atualmente 74,9 anos; Aumento na população de idosos $\geq 60$ anos na última década (2000 a 2010), de 6,7\% para 10,8\%.1 (Sociedade Brasileira de Cardiologia, 2016).

Segundo um estudo realizado por Silva; Oliveira e Pierin (2016) sobre o controle da hipertensão arterial em mulheres e homens: uma análise comparativa foi evidenciada que apesar da presença de fatores desfavoráveis, como menor renda, índice de massa corporal elevado, menos apoio social e maior percentual de transtornos mentais comuns, as mulheres mostravam valores da pressão arterial mais controlado em relação os homens. Tal repercussão pode ser decorrente delas possuírem uma percepção mais clara de sua condição de saúde e, dessa forma, não somente buscarem mais os serviços de saúde, bem como tendem a seguir os tratamentos propostos.

O estudo Corações Brasil mostrou a seguinte distribuição sobre a HA: $10 \%$ na amarela; 26,3\% na parda/mulata; 29,4\% na branca e 34,8 na raça negra. Já o estudo ELSA-Brasil observou uma taxa de 30,3\% em brancos, 38,2 em pardos e 49,3\% em negros (Sociedade Brasileira de Cardiologia 2016).

Adultos sem instrução ou ensino fundamental incompleto (menor índice de escolaridade) apresentaram a maior taxa de HA $(31,1 \%)$. A parcela diminuiu naqueles que completam o ensino fundamental $(16,7 \%)$, mas, em relação às pessoas com superior completo, o índice foi 18,2\% (Scala et al., 2015).

Dados da Pesquisa Nacional da Saúde (PNS) indicam que indivíduos insuficientemente ativos adultos que não atingiram pelo menos 150 minutos semanais de atividade física considerando o lazer, o trabalho e o deslocamento correspondem 46,0\% dos adultos, sendo o percentual significantemente maior entre as mulheres (51,5\%) (Malta et al., 2015). 
O sedentarismo hoje passa a ser compreendido como um problema de saúde pública no século 21, uma pandemia mundial que está relacionada a diversas comorbidades, como hipertensão arterial sistêmica, diabetes mellitus tipo 2, obesidade, síndrome metabólica e dislipidemia entre outras (Aziz, 2014). Dados de diversas pesquisas apontam uma relação linear e direta entre o índice de massa corporal (IMC) e pressão arterial, sendo mais importante para a localização central de gorduras ou obesidade abdominal que a periférica. Homens e mulheres, que apresentam predominantemente a gordura no abdômen e ombro estão associada mais diretamente com doenças cardiovasculares e cerebrovasculares. (Guia De Seguimento Farmacoterapeutico Sobre Hipertension, 2015).

A relação entre uma dieta rica em sódio e sua excreção urinária como fator etiológico primário da HA em humanos é velha. Comprovações epidemiológicas certificam haver uma convergência direta entre a quantidade da ingestão de sódio na dieta e o aumento da prevalência da HA. Populações em consumo de pequenas quantidades sódio praticamente não apresentam hipertensão (Bombing; Francisco; Machado, 2014).

Além do sódio, o cloreto que acompanha o sódio no sal também parece ser um vilão na patogenia da HA. Aumentando-se a ingestão de cloreto de sódio em pacientes sensíveis ao sódio, ocorre amplificação de volume e aumento da pressão. Na entre associação entre a PA e o sódio, estão envolvidos fatores hemodinâmicos e não hemodinâmicos, que induzem mecanismos cardiovasculares adversos (Bombing; Francisco; Machado, 2014).

O tratamento da HAS representa um grande desafio principalmente quando se trata do paciente idoso, visto que se trata de um grupo variado, com múltiplas doenças associadas, problemas cognitivos, risco de queda, polifarmácia, apresenta fragilidade e entre outros motivos, portanto, no idoso as metas terapêuticas devem ser individualizado e acompanhado a fim de garantir qualidade de vida ao paciente (Santos et al., 2019).

A HAS constitui-se um parâmetro de gravidade para o desenvolvimento de doenças cardiovasculares, sendo ainda caracterizado como importante problema de saúde pública. O tratamento da HAS está pautado basicamente medidas medicamentosas ou em terapias não medicamentosas que consiste em estratégias que visam mudar o estilo de vida e levar a uma redução das dosagens ou até mesmo na dispensa dos medicamentos (Gomes; Pagan; Okoshi, 2019).

Estimativas do Ministério da Saúde indica que cerca de 70\% dos homens e 61\% das mulheres com HAS são pacientes obesos. A HAS está diretamente interligada ao peso corporal do paciente. Os pacientes com IMC maior que $30 \mathrm{~kg} / \mathrm{m}^{2}$ tem mais de 50\% chance de apresentar uma pressão arterial descontrolada. Portanto, é importante que o paciente seja educado quanto a sua condição e desempenha mudanças no seu estilo de vida a fim de controlar a HAS. Por esse motivo é importante a prática de exercícios físicos, visto que essa prática auxilia em diversas funções fisiológicas no corpo como diminuir HAS (Burgos et al., 2014).

Segundo a OMS o consumo de sódio é um importante fator para o desenvolvimento da HAS, visto que redução da ingestão de sódio para $2 \mathrm{~g} / \mathrm{dia}$ ou cloreto de sódio para $5 \mathrm{~g} / \mathrm{dia}$, reduz a PA efetivamente em cerca de 2 a $8 \mathrm{mmHg}$. Dados revelam que o consumo diário de sódio no Brasil chega ser $12 \mathrm{~g} / \mathrm{dia}$, o aumento da PA ele está relacionado à propriedade osmótica do sódio que auxilia na retenção de água (Bombing; Francisco; Machado, 2014).

A ingestão de bebidas alcoólicas pode aumenta a PA e o seu consumo em excesso aumenta os riscos de incidências da HAS. Estima-se que a ingestão de $10 \mathrm{~g} /$ dia de bebidas alcoólicas tem consequências sobre a PA em cerca de $1 \mathrm{mmHg}$, sendo que consequentemente a redução do consumo de bebida alcoólica pode ser recomendada como tratamento não farmacológico para o tratamento da HAS (Souza, 2014). 
Tabela 1 - Recomendações de medidas não farmacológicas.

\begin{tabular}{|c|c|c|}
\hline Medida & $\begin{array}{l}\text { Reduçåo aproximada } \\
\text { da PAS/PAD }\end{array}$ & Recomendaçâo \\
\hline Controle do peso & $\begin{array}{l}20-30 \% \text { de diminuiçıo da PA para cada } 5 \% \text { de } \\
\text { perda ponderal' }\end{array}$ & $\begin{array}{l}\text { Manter } \mathrm{IMC}<25 \mathrm{~kg} / \mathrm{m}^{2} \text { até } 65 \text { anos. } \\
\text { Manter } \mathrm{IMC}<27 \mathrm{~kg} / \mathrm{m}^{2} \text { após } 65 \text { anos. } \\
\text { Manter } \mathrm{CA}<80 \mathrm{~cm} \text { nasmulherese }<94 \mathrm{~cm} \text { nos homens }\end{array}$ \\
\hline Padrầo alimentar & Reduçín de $6,7 / 3,5 \mathrm{mmH}^{35}$ & Adotar a dieta DASH \\
\hline $\begin{array}{l}\text { Moderação no consumo de } \\
\text { álcool }\end{array}$ & $\begin{array}{l}\text { Reduço de } 3,31 / 2,04 \mathrm{mmH}_{\mathrm{g}} \text { com a reduçio de } \\
\text { 3-6 para 1-2 doses/dia }\end{array}$ & $\begin{array}{l}\text { Limitar o consumo diário de álcool a } 1 \text { dose nas } \\
\text { mulheres e pesscas com baixo peso e } 2 \text { doses nos } \\
\text { homens }\end{array}$ \\
\hline
\end{tabular}

Fonte: Sociedade Brasileira de Cardiologia (2017 p.38).

Todos os medicamentos utilizados na terapia da HAS deve ser observada a indicação, contra-indicações e além de conhecer as peculiaridades do paciente, visto que quando se trata do paciente idoso existe mais comorbidades associadas ao mesmo paciente. Para que haja essa indicação do medicamento para o paciente hipertenso é necessário levar em consideração vários aspectos como: ser eficaz por via oral; ser bem tolerado; poder ser usado no menor número de tomadas por dia; poder ser usado em associação e entre outros aspectos (Santimaria et al., 2019).

Os diuréticos (DIU) são os medicamentos de primeira escolha para tratamento da HAS em idosos sem comunidades. Os diuréticos são medicamentos de primeira escolha para o combate à HAS e quadros de edema (acúmulo de água resultando em inchaço), bem como outros distúrbios como ICC e IRC. Em grande maioria dos casos os DIU não são utilizados de forma isolados, sempre estão associados ao esquema terapêutico de um paciente. Entretanto, esse tipo de medicamento deve ser evitado ou adequado com horário do paciente, visto que aumenta o fluxo urinário trazendo risco de queda (Moreira; Cipullo; Vilela- Martin, 2013).

Os agentes alfa-agonistas de ação central têm sua ação através de neuroestímulos dos receptores alfa, produzindo efeitos simpatonibitórios, contribuindo para a diminuição da resistência vascular periférica e do débito cardíaco. Os medicamentos representantes desse grupo são a metildopa, clonidina e outros inibidores dos receptores imidazolínicos. Os alfabloqueadores atua em diversos receptores promovendo efeito hipotensor, melhora no metabolismo lipídico e glicídico, sendo amplamente utilizado de forma associada aos medicamentos. Os medicamentos dessa classe são representados pelo doxazosina, prazosina e terazosina (Daniel; Veiga, 2013).

Os betabloqueadores (BB) tem ação nos receptores beta, promovendo a diminuição inicial do débito cardíaco e a liberação de renina. É importante ressaltar que os BB não podem ser indicados para pacientes que tenham asma, visto que tem receptores específicos no pulmão podendo provocar uma broncoconstrição. Já os Bloqueadores dos Canais de Cálcio (BCC) auxiliam na diminuição da resistência vascular periférica por meio do bloqueio dos canais de cálcio dentro das células, sendo grandes representantes dessa classe nifedipina, anlodipino e verapamil (Sociedade Brasileira De Cardiologia, 2017).

Uma das classes mais conhecidas entre os anti-hipertensivos é os Inibidores da Enzima Conversora de Angiotensina (IECA) e Bloqueadores dos Receptores AT1 da Angiotensina 2 (BRA). Os IECAs e BRAs ambos atuam no sistema renina angiotensina aldosterona (SRAA) apresentando diversas funções inibitórias auxiliando no controle da HAS. Um dos grandes representantes da IECA é o Captopril que apresenta como principal efeito adverso à tosse seca acometendo cerca de 5 a $20 \%$ dos pacientes (Lima; Nunes 2019).

O farmacêutico é um profissional extremamente importante para monitorização em saúde, sendo também um dos profissionais da Saúde responsável pelo combate e prevenção da HAS. O farmacêutico participa da farmacoterapia do paciente com HAS realizando atividades simples como o ato da aferição da pressão arterial em farmácias e drogarias, bem como 
realização do acompanhamento da terapia medicamentosa do paciente. A aferição de parâmetros fisiológicos ou bioquímicos pode ser oferecida nas drogarias e farmácias sendo atividade reconhecida como parte da atenção farmacêutica pela resolução da diretoria colegiada (RDC) nº44 de 17 de agosto de 2009 (Toledo et al., 2016).

Em farmácias e drogarias o farmacêutico pode colaborar com o tratamento medicamentoso de um paciente com HAS por meio dos serviços regulamentados pela RDC no 44 de 2009. Para a prestação desse serviço o farmacêutico deve elaborar um Procedimento Operacional Padrão (POP) de acordo com o perfil do paciente que pretende fazer o acompanhamento e especificar os parâmetros com finalidade de dar subsídio à atenção farmacêutica (Toledo et al., 2016).

Segundo Bittencourt e Oliveira (2020) o diagnóstico da HAS é realizado pela detecção de altos e sustentáveis níveis da PA pela medida casual. A verificação da PA deve ser realizada em qualquer tipo de avaliação ou atendimento clínico, seja médico ou de qualquer outra especialidade de profissionais da saúde. O processo de medição da PA é simples, porém nem sempre são realizados seguindo as condutas corretas. Algumas condutas assertivas de medição de PA podem evitar erros, como, preparo apropriado do paciente, uso de técnica padronizada e equipamento de medição calibrado.

Os procedimentos recomendados para a medição da PA, segundo a Sociedade Brasileira de Cardiologia (2017) explicar como funcionará o procedimento para o paciente e deixado em repouso por pelo menos cinco minutos em um ambiente calmo. É importante que o paciente não converse ou se mova durante o procedimento, então quaisquer dúvidas devem ser esclarecidas antes ou após o procedimento, certificando-se de que o paciente não esteja com a bexiga cheia ou tenha praticado exercícios físicos a pelo menos uma hora atrás, ingeriu bebidas alcoólicas, café ou alimentos, e nem que tenha fumado nas últimas meia hora.

Também é importante que o paciente esteja posicionado da maneira correta. O paciente deverá estar sentado, de pernas descruzadas, pés apoiados no chão, dorso recostado na cadeira e relaxado. $\mathrm{O}$ braço deve estar na altura do coração, usando roupas que não sejam justas e apoiaram com a palma da mão voltada para cima e o cotovelo ligeiramente flexionado (Mata; Godoi Filho; Cesarino, 2020).

O farmacêutico tem um importante papel como orientador da terapia medicamentosa do paciente visto seu profissional inteiramente responsável pelo uso racional do medicamento proporcionando orientações que promovam a segurança ao paciente como dosagem certa, paciente certo, horário certo, medicamento certo e entre outros. Visto isso a promoção da educação em saúde para manutenção dos processos de adesão ao tratamento, faz parte do aspecto fundamental do farmacêutico servindo como uma forma de prevenção de complicações cardiovasculares decorrente da HAS (Oliveira; Menezes, 2013).

Outra importante atividade realizada pelo farmacêutico no tratamento da HAS é realizar o acompanhamento farmacoterapêutico. O acompanhamento farmacoterapêutico tem como objetivo garantir o tratamento mais indicado, efetivo, seguro e adequado a esses usuários, sendo assim a atividade do farmacêutico passa a ser deslocada do produto para o serviço e do medicamento para o paciente, valorizando os problemas de saúde e buscando resolvê-los através de intervenções farmacêuticas (Aires; Marchiorato, 2010).

O acompanhamento farmacoterapeutico deve ser realizado de maneira eficiente e buscando sempre a melhoria na qualidade de vida do paciente, uma referência de modelo preconizado é o modelo espanhol de acompanhamento método dáder. O método dáder se baseia na obtenção do histórico farmacoterapêutico do paciente, assim como os problemas de saúde apresentado pelo usuário e os medicamentos utilizados pelo mesmo. Também é realizada avaliação do seu estado atual ou em uma determinada data a fim de identificar e resolver possíveis problemas relacionados com os medicamentos (PRM). O Segundo Consenso de Granada estabelece uma classificação para os possíveis PRM em seis categorias em que são avaliados segundo a sua necessidade efetividade e segurança, segundo demonstrado na Tabela 2 (Oliveira; Menezes, 2013). 
Tabela 2 - Classificação dos PRM, a partir das características de necessidade, efetividade e segurança.

\begin{tabular}{l} 
Necessidade \\
\hline $\begin{array}{l}\text { PRM 1: O paciente apresenta um problema de saúde por não utilizar a } \\
\text { farmacoterapia que necessita }\end{array}$ \\
\hline $\begin{array}{l}\text { PRM 2: O paciente apresenta um problema de saúde por utilizar um medicamento } \\
\text { que não necessita }\end{array}$ \\
\hline $\begin{array}{l}\text { PRM 3: O paciente apresenta um problema de saúde por uma inefetividade nāo } \\
\text { quantitativa da farmacoterapia }\end{array}$ \\
\hline $\begin{array}{l}\text { PRM 4: O paciente apresenta um problema de saúde por uma inefetividade } \\
\text { quantitativa da farmacoterapia }\end{array}$ \\
\hline $\begin{array}{l}\text { PRM 5: O paciente apresenta um problema de saúde por uma insegurança não } \\
\text { quantitativa de um medicamento }\end{array}$ \\
\hline $\begin{array}{l}\text { PRM 6: O paciente apresenta um problema de saúde por uma insequrança } \\
\text { quantitativa de um medicamento }\end{array}$ \\
\hline
\end{tabular}

Fonte: Ferreira et al. (2013 p, 4).

Como já citado anteriormente para se alcançar o sucesso no tratamento do paciente hipertenso é importante considerar diversos fatores: a influência da HAS em todo o organismo do paciente, aferições rotineiras da PA com o objetivo de monitorar os níveis de segurança, efetividade e necessidade da farmacoterapia e da terapia não medicamentosa, assim como avaliação de outros medicamentos em uso pelo pacientes e possíveis interações medicamentosas (Marchesan, 2018).

A presença do profissional farmacêutico em todo âmbito do cuidado em pacientes hipertensos é extremamente importante e depende unicamente da aderência ao tratamento. Pelo farmacêutico seu profissional da Saúde mais próximo da comunidade por meio de farmácias e drogarias, o farmacêutico pode realizar um acompanhamento do paciente auxiliando de forma positiva no acompanhamento dos parâmetros físicos e biológicos do paciente, bem como auxiliando no aconselhamento a respeito da terapia medicamentosa e proporcionando o uso racional de medicamento (Oliveira; Menezes, 2013).

\section{Considerações Finais}

Neste trabalho abordamos sobre a hipertensão arterial como um problema de saúde que afeta grande parte da população brasileira, em especial idosa e adulta. O surgimento da hipertensão está associado a diversos fatores como idade, fatores genéticos, obesidade, alimentação desequilibrada, entre outros motivos. É notável a necessidade de adoção de medidas não farmacológicas para o tratamento da hipertensão arterial.

O tratamento da HA está associado ao uso de diversos anti-hipertensivos e diuréticos, sendo também importante adotar um novo estilo de vida. A escolha do medicamento dependerá de diversos fatores clínicos do paciente, também a resposta do mesmo. O farmacêutico assume importantíssimo no cuidado e acompanhamento do paciente hipertenso por meio da aferição de pressão arterialem farmácias e drogarias. Fora isso o farmacêutico contribui por meio do acompanhamento farmacoterapêutico dois medicamentos auxiliando na adesão e nouso racional dos medicamentos pelo paciente. 


\section{Referências}

Aires, C. C. N. D. F., \& Marchiorato, L. (2010). Acompanhamento farmacoterapêutico a hipertensos e diabéticos na unidade de saúde Tereza Barbosa: análise de caso. Revista Brasileira de Farmácia Hospitalar e Serviços de Saúde, 1(1).

Aziz, J. L. (2014). Sedentarismo e hipertensão arterial. Revista Brasileira de Hipertensão, 21(2), 75-82.

Bombig, M. T. N., Francisco, Y. A., \& Machado, C. A. (2014). A importância do sal na origem da hipertensão. Revista Brasileira de Hipertensão, 21(2), 6367.

Brandão, A. A., Magalhães, M. E. C., Ávila, A., Tavares, A., Machado, C. A., Campana, É. M. G., \& Sampaio, R. (2010). Conceituação, epidemiologia e prevenção primária. Brazilian Journal of Nephrology, 32, 1-4.

Coelho, J. C., Ferretti-Rebustini, R. E. L., Suemoto, C. K., Leite, R. E. P., Jacob-Filho, W., \& Pierin, A. M. G. (2019). A hipertensão arterial é causa subjacente de morte avaliada na autópsia de indivíduos. Revista da Escola de Enfermagem da USP, 53.

Daniel, A. C. Q. G., \& Veiga, E. V. (2013). Fatores que interferem na adesão terapêutica medicamentosa em hipertensos. Einstein (São Paulo), 11(3), 331-337.

de Oliveira Silva, D., de Sousa, D. M., Santana, G. C. M., Meneguelli, A. Z., \& de Oliveira Salvi, J. (2020). Atenção Farmacêutica na Melhor Idade: Um Relato de Experiência na Unidade Básica Juscelino Cardoso em Ji-Paraná-RO. Revista Enfermagem e Saúde Coletiva-REVESC, 5(1), 75-82.

de Souza, D. D. S. M. (2014). Álcool e hipertensão. Aspectos epidemiológicos, fisiopatológicos e clínicos. Rev Bras Hipertens vol, 21(2), 83-86.

Gomes, M. J., Pagan, L. U., \& Okoshi, M. P. (2019). Tratamento Não Medicamentoso das Doenças Cardiovasculares| Importância do Exercício Físico. Arquivos Brasileiros de Cardiologia, 113(1), 9-10.

Lima, M. M., \& Nunes, C. P. (2019). Eficácia Do Uso De Ieca/Bra Na Diminuição Da Mortalidade Pós Infarto Do Miocárdio Em Hipertensos Com Complicações Cardiovasculares. Revista de Medicina de Família e Saúde Mental, 1(1).

Malta, D. C., Andrade, S. S. C. D. A., Stopa, S. R., Pereira, C. A., Szwarcwald, C. L., Silva Júnior, J. B. D., \& Reis, A. A. C. D. (2015). Brazilian lifestyles: National Health Survey results, 2013. Epidemiologia e Serviços de Saúde, 24, 217-226.

Marchesan, L. B. (2018). Critérios ACC/AHA 2017 para diagnóstico de hipertensão arterial em pacientes com a síndrome dos ovários policísticos e associação com variáveis metabólicas.

Maricato, L. I. G. (2018, November). Consultório Farmacêutico-Serviços farmacêuticos para pacientes polimedicados atendidos na Farmácia Comunitária Vital Brazil. In $16^{\circ}$ ENEX.

Marin, N. S., Santos, M. F. D., \& Moro, A. D. S. (2016). Perception of hypertensive patients about their non-adherence to the use of medication. Revista da Escola de Enfermagem da USP, 50(SPE), 61-67.

Mata, J. G. F., de Godoi Filho, M. B., \& Bernardi Cesarino, C. (2020). Adesão Ao Tratamento Medicamentoso De Adultos Autorreferidos Com Diagnóstico De Hipertensão. Saúde e Pesquisa, 13(1).

Mion Jr, D., Pierin, A. M. G., \& Guimarães, A. (2001). Tratamento da hipertensão arterial-respostas de médicos brasileiros a um inquérito. Revista da Associação Médica Brasileira, 47(3), 249-254.

Moreira, G. C., Cipullo, J. P., \& Vilela-Martin, J. F. (2013). Existem diferenças entre os diversos diuréticos. Rev Bras Hipertens, $20(2)$, 55-62.

Malachias, M. V. B., Plavnik, F. L., Machado, C. A., Malta, D., Scala, L. C. N., \& Fuchs, S. (2016). $7^{\mathrm{a}}$ Diretriz Brasileira de Hipertensão Arterial: Capítulo 1Conceituação, Epidemiologia e Prevenção Primária. Arquivos Brasileiros de Cardiologia, 107(3), 1-6.

Santimaria, M. R., Borim, F. S. A., Leme, D. E. D. C., Neri, A. L., \& Fattori, A. (2019). Falha no diagnóstico e no tratamento medicamentoso da hipertensão arterial em idosos brasileiros-Estudo FIBRA. Ciência \& Saúde Coletiva, 24, 3733-3742.

Santos, D. M. S., Prado, B. S., Oliveira, C. C. D. C., \& Almeida-Santos, M. A. (2019). Prevalência da Hipertensão Arterial Sistêmica em Comunidades Quilombolas do Estado de Sergipe, Brasil. Arquivos Brasileiros de Cardiologia, 113(3), 383-390.

Sarno, F., Bittencourt, C. A. G., \& Oliveira, S. A. D. (2020). Perfil de pacientes com hipertensão arterial e/ou diabetes mellitus de unidades de Atenção Primária à Saúde. Einstein (Sao Paulo).18.

Scala, L. C., Magalhães, L. B., \& Machado, A. (2015). Epidemiologia da hipertensão arterial sistêmica. Sociedade Brasileira de Cardiologia. Livro Texto da Sociedade Brasileira de Cardiologia.

Silva, S. S. B. E. D., Oliveira, S. D. F. D. S. B. D., \& Pierin, A. M. G. (2016). El control de la hipertensión arterial en mujeres y hombres: un análisis comparativo. Revista da Escola de Enfermagem da USP, 50(1), 50-58.

Souza, D. D. S. M. (2014). Álcool e hipertensão. Aspectos epidemiológicos, fisiopatológicos e clínicos. Rev Bras Hipertens vol, 21(2), 83-86.

Toledo, T. R., et al. Abordagem farmacoepidemiológica dos pacientes hipertensos frequentadores de uma drogaria de um município de Minas Gerais. Revista Científica Da Faminas, 9. 